\title{
A dose-dependent perturbation in cardiac energy metabolism is linked to radiation-induced ischemic heart disease in Mayak nuclear workers
}

\author{
Omid Azimzadeh ${ }^{1}$, Tamara Azizova ${ }^{2}$, Juliane Merl-Pham ${ }^{3}$, Vikram Subramanian ${ }^{1}$, \\ Mayur V. Bakshi ${ }^{1}$, Maria Moseeva ${ }^{2}$, Olga Zubkova ${ }^{2}$, Stefanie M. Hauck ${ }^{3}$, Nataša \\ Anastasov $^{1}$, Michael J. Atkinson ${ }^{1,4}$, Soile Tapio ${ }^{1}$ \\ ${ }^{1}$ Helmholtz Zentrum München-German Research Center for Environmental Health (GmbH), Institute of Radiation Biology, \\ Neuherberg, Germany \\ ${ }^{2}$ Southern Urals Biophysics Institute, Russian Federation, Ozyorsk, Russia \\ ${ }^{3}$ Helmholtz Zentrum München-German Research Center for Environmental Health (GmbH), Research Unit Protein Science, \\ Munich, Germany \\ ${ }^{4}$ Chair of Radiation Biology, Technical University of Munich, Munich, Germany
}

Correspondence to: Soile Tapio, email: soile.tapio@helmholtz-muenchen.de

Keywords: ionising radiation, proteomics, heart disease, PPAR alpha, mitochondrial dysfunction

Received: April 08, 2016 Accepted: June 17, $2016 \quad$ Published: July 06, 2016

\section{ABSTRACT}

Epidemiological studies show a significant increase in ischemic heart disease (IHD) incidence associated with total external gamma-ray dose among Mayak plutonium enrichment plant workers. Our previous studies using mouse models suggest that persistent alteration of heart metabolism due to the inhibition of peroxisome proliferator-activated receptor (PPAR) alpha accompanies cardiac damage after high doses of ionising radiation. The aim of the present study was to elucidate the mechanism of radiation-induced IHD in humans. The cardiac proteome response to irradiation was analysed in Mayak workers who were exposed only to external doses of gamma rays. All participants were diagnosed during their lifetime with IHD that also was the cause of death. Label-free quantitative proteomics analysis was performed on tissue samples from the cardiac left ventricles of individuals stratified into four radiation dose groups ( $0 \mathrm{~Gy},<100 \mathrm{mGy}, 100-500 \mathrm{mGy}$, and $>500 \mathrm{mGy}$ ). The groups could be separated using principal component analysis based on all proteomics features. Proteome profiling showed a dose-dependent increase in the number of downregulated mitochondrial and structural proteins. Both proteomics and immunoblotting showed decreased expression of several oxidative stress responsive proteins in the irradiated hearts. The phosphorylation of transcription factor PPAR alpha was increased in a dose-dependent manner, which is indicative of a reduction in transcriptional activity with increased radiation dose. These data suggest that chronic external radiation enhances the risk for IHD by inhibiting PPAR alpha and altering the expression of mitochondrial, structural, and antioxidant components of the heart.

\section{INTRODUCTION}

Mayak Production Association (PA), located $150 \mathrm{~km}$ south-east of Ekaterinburg, is one of the biggest nuclear facilities in the Russian Federation. Individual dosimetric monitoring of external exposure performed at Mayak PA showed that the total external gamma-ray doses ranged widely from below $100 \mathrm{mGy}$ to more than $5 \mathrm{~Gy}$, with $32.6 \%$ of the workers having a total dose greater than 1 Gy [1].
Epidemiological studies in this cohort showed a significant increase in ischemic heart disease (IHD) incidence associated with total external gamma-ray dose after correction for multiple competing factors such as smoking and alcohol consumption [1-3]. The risk estimates for IHD in relation to chronic external radiation dose are generally compatible with those reported in other large occupational studies and the Japanese A-bomb survivors [4]. 
Mitochondrial dysfunction plays a key role in the pathogenesis of IHD [5]. A high rate of mitochondrial catabolism of carbohydrates and fatty acids is crucial for furnishing the energy supply necessary for heart function [6]. Under normal conditions the adult heart relies mostly on fatty acids for this energy production via the oxidative phosphorylation (OXPHOS) process, with only $10 \%$ to $30 \%$ of total ATP being derived from glucose [7]. However, a normal heart can easily switch between fatty acids and glucose for ATP production, depending on energy demand and substrate availability [8]. In pathological conditions such as IHD this flexibility is lost and either superseded by a preference for glucose over fat [9] or an overall reduction of mitochondrial oxidative metabolism independent of the energy source [10]. Both scenarios are associated with the reduction in the level of active peroxisome proliferator-activated receptor (PPAR) alpha in cardiac ventricles [11]. PPAR alpha functions as a key regulator of cardiac metabolism and is essential for fatty acid oxidation [6].

We have previously shown that local heart irradiation in mice persistently decreases the respiratory capacity of cardiac mitochondria $[12,13]$, reduces their number, and results in damage to the cristae structure [14]. Importantly, the activity of the transcription factor PPAR alpha is reduced by a dose-dependent increase in phosphorylation [14].

Although mouse models are widely used to study cardiac disease, there are functional differences between mouse and human hearts [15]. Infarction is virtually unknown in mice, probably due to their short life span, and differences in the heart physiology and diet. Even though mouse models have led to important observations on the causes of radiation-induced IHD, the question of their clinical relevance remains.

The aim of the present study was to examine whether alteration in cardiac metabolism, and its key regulator PPAR alpha, contribute to radiation-induced IHD in man. Here, we investigated human left ventricle proteome profiles in Mayak workers who had been occupationally exposed to different cumulative doses of external gamma rays. All participants had previously been diagnosed with IHD that also was the primary cause of death $[1,16]$. The proteomic analysis revealed a dose-dependent series of alteration in the levels of proteins involved in the left ventricle function and structure. These include proteins critical for mitochondrial energy metabolism and cardiac cytoskeleton. A significant inactivation of PPAR alpha by phosphorylation was observed in the highest dose group (> $500 \mathrm{mGy}$ ). The present study provides, for the first time, a proteomics signature of radiation-induced human heart ischemia. This is coherent with the observations made using irradiated mice upon the radiation dose.

\section{RESULTS}

\section{Chronic irradiation alters the heart proteome in a dose-dependent manner}

Global proteomics analysis identified 1,281 proteins in total (Supplementary Table S1). Of the quantified proteins, 101, 225 and 431 proteins were significantly changed in expression ( 2 unique peptides; fold change $\geq 1.30$ or $\leq 0.77$; $\mathrm{q}<0.05$ ) after exposure to doses of $<100 \mathrm{mGy}, 100-500 \mathrm{mGy}$ and $>500 \mathrm{mGy}$, respectively. This indicated a dose-dependent increase in the number of deregulated proteins (Supplementary Tables S2-S4), as seen in irradiated mouse heart models [14]. A large number (72) of deregulated proteins were shared between all three irradiated groups compared to the control (Table 1). The majority of these shared proteins belonged to mitochondria (24 proteins) or cytoskeleton (13 proteins).

To investigate differences in the proteome profiles between the different dose groups, a PCA based on all proteomics features was performed. Control and irradiated samples clustered into four groups according to the dose (Figure 1). The distance between the cluster that represents the control group and the clusters representing the irradiated groups increased with increasing dose. Even though the workers exposed to the highest dose $(>500$ mGy) were generally older than the members of other groups, the PCA did not show any clustering based on age. Similarly, no clustering was observed based on smoking status or index, alcohol consumption, or body mass index (Supplementary Table S10).

Some outliers were identified in each irradiated group, namely donors 3, 25 and 46 (Figure 1). Sample number 25 , belonging to the group $<100 \mathrm{mGy}$, was exposed to the very low dose of $6 \mathrm{mGy}$, and unsurprisingly showed proteomics features that were more similar to those of the control group. Sample number 3, belonging to the dose group of 100-500 mGy, was exposed to the dose of $114 \mathrm{mGy}$ and showed similarity with the group of $<100$ mGy. Sample number 46, a member of the dose group 100-500 mGy, was exposed to the dose of 483 $\mathrm{mGy}$, and was placed in close proximity to the group exposed to the highest dose (> $500 \mathrm{mGy}$ ) (Figure 1 and Supplementary Table S10). These deviations strengthen the evidence for a dose-response relationship.

A detailed analysis of functional interactions and biological pathways was performed using IPA (http://www. INGENUITY.com) (Supplementary Tables S5 and S6). Mitochondrial dysfunction and metabolic impairment were indicated in all irradiated groups compared to the control group (Figure 2A). A dose-dependent reduction was found in the expression of proteins of the respiratory complexes I, III and V. The complexes II and IV were affected only 
Table 1: Significantly deregulated proteins shared in all radiation dose groups

\begin{tabular}{|c|c|c|c|c|c|c|}
\hline \# & Symbol & Entrez Gene Name & ratio & ratio & ratio & GO - Molecular function \\
\hline & & & $\begin{array}{l}<100 \mathrm{mGy} / \\
\text { control }\end{array}$ & $\begin{array}{l}100-500 \\
\mathrm{mGy} / \text { controls }\end{array}$ & $\begin{array}{l}>500 \mathrm{mGy} / \\
\text { controls }\end{array}$ & \\
\hline 1 & ACAT1 & acetyl-CoA acetyltransferase 1 & 0.77 & 0.68 & 0.42 & metabolic activity (GO:0003824) \\
\hline 2 & AHSG & alpha-2-HS-glycoprotein & 0.47 & 0.63 & 0.33 & metabolic activity (GO:0003824) \\
\hline 3 & AIFM1 & apoptosis-inducing factor, mitochondrion-associated, 1 & 0.65 & 0.62 & 0.40 & antioxidant activity (GO:0016209) \\
\hline 4 & AK2 & adenylate kinase 2 & 0.74 & 0.76 & 0.48 & metabolic activity (GO:0003824) \\
\hline 5 & ALDOA & aldolase A, fructose-bisphosphate & 0.63 & 0.63 & 0.42 & metabolic activity (GO:0003824) \\
\hline 6 & ANXA11 & annexin A11 & 0.77 & 0.73 & 0.53 & structural molecule activity (GO:0005198) \\
\hline 7 & ATP5B & ATP synthase, $\mathrm{H}+$ transporting, beta & 0.62 & 0.62 & 0.38 & metabolic activity (GO:0003824) \\
\hline 8 & CCT3 & chaperonin containing TCP1, subunit 3 (gamma) & 0.65 & 0.76 & 0.52 & ATP binding (GO:0005524) \\
\hline 9 & CHCHD3 & coiled-coil-helix-coiled-coil-helix domain containing 3 & 0.46 & 0.6 & 0.34 & structural molecule activity (GO:0005198) \\
\hline 10 & CKM & creatine kinase, muscle & 0.66 & 0.65 & 0.38 & metabolic activity (GO:0003824) \\
\hline 11 & COQ9 & coenzyme Q9 & 0.73 & 0.55 & 0.38 & metabolic activity (GO:0003824) \\
\hline 12 & DBI & GABA receptor modulator, acyl-CoA binding protein & 0.73 & 0.68 & 0.41 & metabolic activity (GO:0003824) \\
\hline 13 & DECR1 & 2,4-dienoyl CoA reductase 1, mitochondrial & 0.7 & 0.57 & 0.39 & metabolic activity (GO:0003824) \\
\hline 14 & DLD & dihydrolipoamide dehydrogenase & 0.74 & 0.64 & 0.51 & metabolic activity (GO:0003824) \\
\hline 15 & ECI1 & enoyl-CoA delta isomerase 1 & 0.7 & 0.65 & 0.44 & metabolic activity (GO:0003824) \\
\hline 16 & FH & fumarate hydratase & 0.71 & 0.61 & 0.40 & metabolic activity (GO:0003824) \\
\hline 17 & GLRX5 & glutaredoxin 5 & 0.52 & 0.5 & 0.37 & antioxidant activity (GO:0016209) \\
\hline 18 & HADH & hydroxyacyl-CoA dehydrogenase & 0.67 & 0.58 & 0.37 & metabolic activity (GO:0003824) \\
\hline 19 & HNRNPA1L2 & heterogeneous nuclear ribonucleoprotein A1-like 2 & 0.77 & 0.73 & 0.56 & nucleotide binding (GO:0000166) \\
\hline 20 & HNRNPA2B1 & heterogeneous nuclear ribonucleoprotein A2/B1 & 0.77 & 0.77 & 0.44 & nucleotide binding (GO:0000166) \\
\hline 21 & HSD17B10 & hydroxysteroid (17-beta) dehydrogenase 10 & 0.76 & 0.66 & 0.42 & metabolic activity (GO:0003824) \\
\hline 22 & LDHB & lactate dehydrogenase B & 0.78 & 0.6 & 0.49 & metabolic activity (GO:0003824) \\
\hline 23 & LGALS1 & lectin, galactoside-binding, soluble, 1 & 0.59 & 0.53 & 0.32 & nucleotide binding (GO:0000166) \\
\hline 24 & LGALS3 & lectin, galactoside-binding, soluble, 3 & 0.48 & 0.49 & 0.29 & nucleotide binding (GO:0000166) \\
\hline 25 & $\mathrm{MCCC} 1$ & methylcrotonoyl-CoA carboxylase 1 (alpha) & 0.62 & 0.52 & 0.39 & ATP binding (GO:0005524) \\
\hline 26 & MCEE & methylmalonyl CoA epimerase & 0.64 & 0.63 & 0.47 & metabolic activity (GO:0003824) \\
\hline 27 & MDH2 & malate dehydrogenase 2, NAD (mitochondrial) & 0.73 & 0.68 & 0.44 & metabolic activity (GO:0003824) \\
\hline 28 & ME3 & malic enzyme 3, NADP $(+)$-dependent, mitochondrial & 3.8 & 6.21 & 4.45 & metabolic activity (GO:0003824) \\
\hline 29 & MECR & mitochondrial trans-2-enoyl-CoA reductase & 0.62 & 0.55 & 0.38 & metabolic activity (GO:0003824) \\
\hline 30 & MYBPC3 & myosin binding protein $\mathrm{C}$, cardiac & 0.74 & 0.57 & 0.45 & structural molecule activity (GO:0005198) \\
\hline 31 & MYH10 & myosin, heavy chain 10 , non-muscle & 2.16 & 2.91 & 1.92 & structural molecule activity (GO:0005198) \\
\hline 32 & MYH11 & myosin, heavy chain 11 , smooth muscle & 0.45 & 0.56 & 0.52 & structural molecule activity (GO:0005198) \\
\hline 33 & MYL2 & myosin, light chain 2 , regulatory, cardiac, slow & 0.63 & 0.65 & 0.40 & structural molecule activity (GO:0005198) \\
\hline 34 & MYL3 & myosin, light chain 3, alkali; ventricular, skeletal, slow & 0.67 & 0.65 & 0.41 & structural molecule activity (GO:0005198) \\
\hline 35 & MYL6 & myosin light chain 6,smooth muscle and non-muscle & 0.54 & 0.68 & 0.38 & structural molecule activity (GO:0005198) \\
\hline 36 & MYOM1 & myomesin 1 & 0.76 & 0.58 & 0.44 & structural molecule activity (GO:0005198) \\
\hline 37 & MYOM2 & myomesin 2 & 0.7 & 0.51 & 0.39 & structural molecule activity (GO:0005198) \\
\hline 38 & NDUFA3 & NADH dehydrogenase (ubiquinone) 1 alpha & 0.41 & 0.56 & 0.29 & metabolic activity (GO:0003824) \\
\hline 39 & NID1 & nidogen 1 & 0.69 & 0.67 & 0.42 & extracellular matrix binding (GO:0050840) \\
\hline 40 & NMT1 & N-myristoyltransferase 1 & 3.4 & 16.63 & 23.75 & apoptotic activity (GO:0006915) \\
\hline 41 & NPM1 & nucleolar phosphoprotein B23, numatrin & 0.72 & 0.77 & 0.44 & histone binding (GO:0042393) \\
\hline 42 & PARK7 & protein deglycase DJ-1 & 0.73 & 0.72 & 0.46 & $\begin{array}{l}\text { nucleic acid binding transcription factor } \\
\text { activity (GO:0001071) }\end{array}$ \\
\hline 43 & PCMT1 & protein-L-isoaspartate (D-aspartate) O-methyltransferase & 0.71 & 0.68 & 0.53 & metabolic activity (GO:0003824) \\
\hline 44 & PDHA1 & pyruvate dehydrogenase (lipoamide) alpha 1 & 0.77 & 0.63 & 0.47 & metabolic activity (GO:0003824) \\
\hline 45 & PDHB & pyruvate dehydrogenase (lipoamide) beta & 0.76 & 0.56 & 0.46 & metabolic activity (GO:0003824) \\
\hline 46 & PGAM2 & phosphoglycerate mutase 2 (muscle) & 0.7 & 0.55 & 0.36 & metabolic activity (GO:0003824) \\
\hline 47 & PGK1 & phosphoglycerate kinase 1 & 0.67 & 0.59 & 0.40 & metabolic activity (GO:0003824) \\
\hline
\end{tabular}




\begin{tabular}{|c|c|c|c|c|c|c|}
\hline 48 & PGM1 & phosphoglucomutase 1 & 0.7 & 0.48 & 0.35 & metabolic activity (GO:0003824) \\
\hline 49 & PKM & pyruvate kinase, muscle & 0.73 & 0.56 & 0.38 & metabolic activity (GO:0003824) \\
\hline 50 & PLEC & plectin & 0.73 & 0.64 & 0.43 & structural molecule activity (GO:0005198) \\
\hline 51 & PPP1CB & protein phosphatase 1 , beta isozyme & 1.61 & 2.09 & 1.62 & structural molecule activity (GO:0005198) \\
\hline 52 & PRDX3 & peroxiredoxin 3 & 0.76 & 0.73 & 0.49 & antioxidant activity (GO:0016209) \\
\hline 53 & PRDX5 & peroxiredoxin 5 & 0.74 & 0.64 & 0.40 & antioxidant activity (GO:0016209) \\
\hline 54 & PRDX6 & peroxiredoxin 6 & 0.74 & 0.72 & 0.47 & antioxidant activity (GO:0016209) \\
\hline 55 & PRKAR1A & protein kinase, cAMP-dependent, regulatory, type I, A & 0.73 & 0.65 & 0.50 & cAMP binding (GO:0030552) \\
\hline 56 & PSMA5 & proteasome (prosome, macropain) subunit, alpha type, 5 & 0.71 & 0.67 & 0.41 & protein polyubiquitination (GO:0000209) \\
\hline 57 & PSMA6 & proteasome (prosome, macropain) subunit, alpha type, 6 & 0.64 & 0.62 & 0.46 & protein polyubiquitination (GO:0000209) \\
\hline 58 & PTRF & polymerase I and transcript release factor & 0.65 & 0.65 & 0.46 & poly(A) RNA binding (GO:0044822) \\
\hline 59 & PYGM & phosphorylase, glycogen, muscle & 0.54 & 0.49 & 0.24 & metabolic activity (GO:0003824) \\
\hline 60 & RPS27A & ribosomal protein $\mathrm{S} 27 \mathrm{a}$ & 0.74 & 0.62 & 0.44 & structural molecule activity (GO:0005198) \\
\hline 61 & SDPR & serum deprivation response & 0.71 & 0.75 & 0.48 & protein kinase $\mathrm{C}$ binding (GO:0005080) \\
\hline 62 & SOD2 & superoxide dismutase 2 , mitochondrial & 0.73 & 0.61 & 0.42 & antioxidant activity (GO:0016209) \\
\hline 63 & SPTA1 & spectrin, alpha, erythrocytic 1 & 4.36 & 4.62 & 4.87 & structural molecule activity (GO:0005198) \\
\hline 64 & SUCLG1 & succinate-CoA ligase, alpha subunit & 0.72 & 0.74 & 0.45 & metabolic activity (GO:0003824) \\
\hline 65 & SUCLG2 & succinate-CoA ligase, beta subunit & 0.66 & 0.67 & 0.42 & metabolic activity (GO:0003824) \\
\hline 66 & TOM1L2 & target of myb1 like 2 membrane trafficking protein & 1.8 & 1.83 & 1.50 & metabolic activity (GO:0003824) \\
\hline 67 & TPM2 & tropomyosin 2 (beta) & 0.49 & 0.5 & 0.33 & structural molecule activity (GO:0005198) \\
\hline 68 & TUBA8 & tubulin, alpha 8 & 0.55 & 0.56 & 0.38 & structural molecule activity (GO:0005198) \\
\hline 69 & TXN & thioredoxin & 0.69 & 0.54 & 0.38 & antioxidant activity (GO:0016209) \\
\hline 70 & UQCR10 & ubiquinol-cytochrome $\mathrm{c}$ reductase, subunit $\mathrm{X}$ & 0.56 & 0.46 & 0.37 & metabolic activity (GO:0003824) \\
\hline 71 & UQCRC2 & ubiquinol-cytochrome $\mathrm{c}$ reductase core protein II & 0.63 & 0.57 & 0.39 & metabolic activity (GO:0003824) \\
\hline 72 & VCAN & versican & 3.05 & 4.11 & 2.31 & structural molecule activity (GO:0005198) \\
\hline
\end{tabular}

The accession number, protein ID, full name and fold change after exposure to $<100 \mathrm{mGy}, 100-500 \mathrm{mGy}$ or $>500 \mathrm{mGy}$ is shown for each protein.

Principal Components Analysis

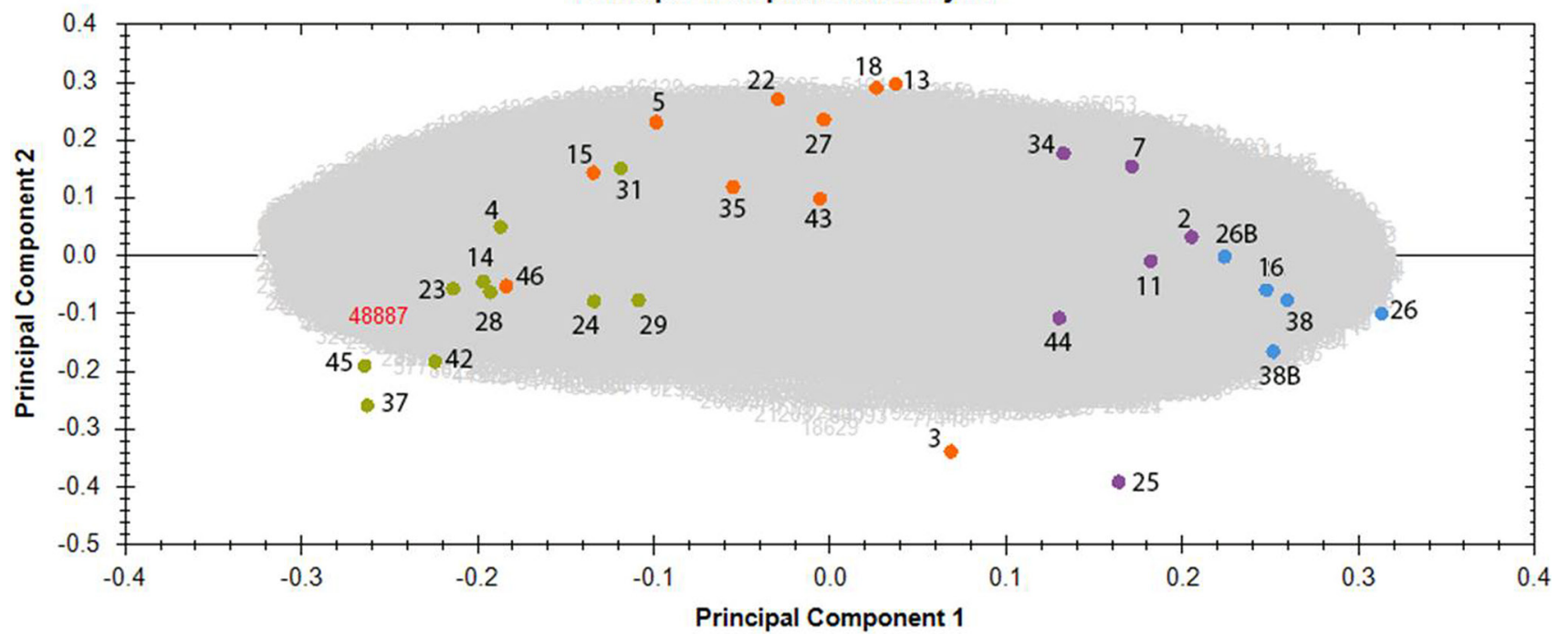

Figure 1: Principal component analysis (PCA) based on all proteomic features in the left ventricle of sample donors in different dose groups. The PCA used features with charges +2 to +7 resulting in PC1 and PC2 as follows: PC1 23.65\% and PC2 8.36\%. The control samples with the corresponding donor number are represented as blue spots, the samples exposed to $<100 \mathrm{mGy}$ in purple, the samples exposed to 100-500 mGy in orange and the samples exposed to $>500 \mathrm{mGy}$ in green. Samples number 26 and 38 were run as 2 technical replicates and are indicated as $26,26 \mathrm{~B}$ and $38,38 \mathrm{~B}$. Detailed information of the sample donors and the exact doses are given in Supplementary Table S10. The analysis was performed using the Progenesis QI software (http://www.nonlinear.com). 
in the two high-dose groups (Figure 2B). The number of deregulated mitochondrial proteins increased with the radiation dose (Figure 2C-2E and Supplementary Table S6).

Several proteins belonging to energy production pathways associated with fatty acid oxidation (lipid metabolism, Krebs cycle) were downregulated by irradiation (Figure 2A and Supplementary Table S6). Also several enzymes in the glycolysis pathway were downregulated (Supplementary Figure S1 and Supplementary Table S6), suggesting a general depletion of energy supply, rather than a glucose/lipid switch.

In addition, the number of deregulated proteins belonging to actin cytoskeleton or calcium signalling was increased in a dose-dependent manner (Supplementary Figure S2 and Supplementary Table S6). The majority of significantly altered proteins were associated with heart diseases including left ventricle dysfunction and heart hypertrophy (Supplementary Figure S3 and Supplementary Table S7).

\section{Immunoblotting confirms radiation-induced downregulation of structural and antioxidant proteins}

Consistent with the proteomics data, immunoblotting showed markedly decreased levels of the antioxidant defence proteins peroxiredoxin 5 (PRDX 5), and superoxide dismutase 2 (SOD2) after irradiation (Figure 3). The expression of transcription factor $\mathrm{Nrf} 2$, the central regulator of the antioxidative response, was significantly downregulated in the highest dose group (Figure 3). Significantly reduced expression of structural proteins myosin light chain 2 (MYL2), tropomyosin 2 (TPM2) and troponin T (TNNT2) was found in the highest dose group (Figure 3).

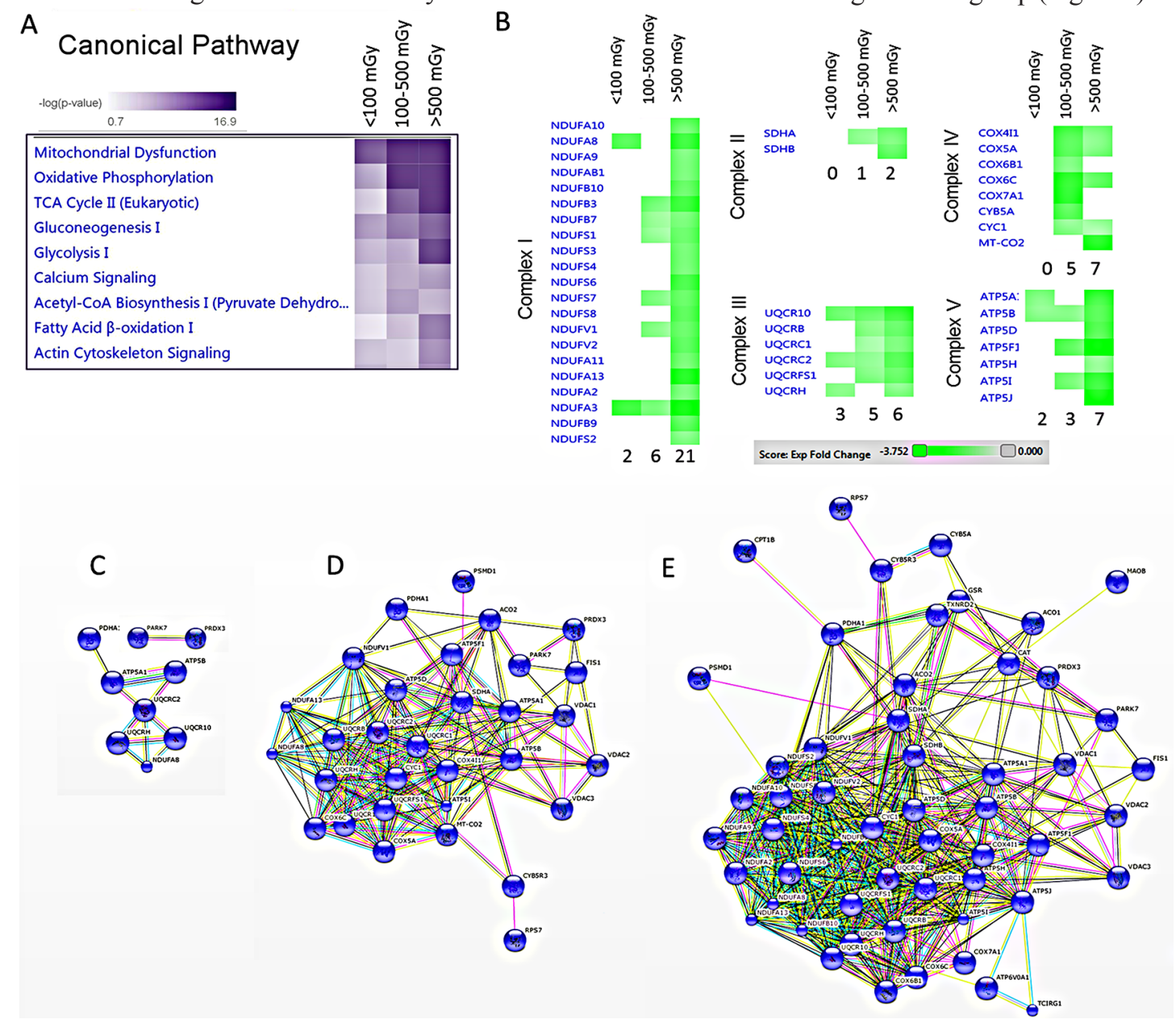

Figure 2: Pathway and network analysis of significantly differentially expressed mitochondrial proteins. A dose-dependent alteration is observed in the pathways involved in the energy production. The pathway scores are displayed using a purple colour gradient, where darker purple corresponds to higher scores (increased statistical significance). The score is the negative log of the $p$-value derived from the Fisher's Exact test. By default, the rows (pathways) with the highest total score across the set of observations are sorted to the top (A). Heat map for the expression values of differentially expressed OXPHOS proteins between dose groups is displayed using a green colour gradient for downregulated proteins, where dark green corresponds to large downregulation. The numbers shows how many proteins were deregulated in each subunit (B) (http://www.INGENUITY.com). Protein-protein interaction analysis of the significantly differentially expressed proteins showing the networks of deregulated mitochondrial proteins in the dose groups $<100 \mathrm{mGy}(\mathbf{C})$, 100-500 mGy (D) and $>500 \mathrm{mGy}$ (E) (http://string-db.org). 


\section{Irradiation enhances protein oxidation}

As the proteomics and immunoblotting data indicated alterations in the oxidative stress response, the level of protein carbonylation (protein oxidation marker) was measured in the pooled samples from each of the different dose groups. A significant increase in protein carbonylation was found in the highest dose group compared to the control (Figure 4A).

\section{Cardiac miRNAs are altered in irradiated hearts}

MicroRNAs miR-21 and miR-146a are potential biomarkers of heart disease [17-19]. The expression of miR-21 and miR-146a was significantly upregulated in the highest dose group compared to the control and lower dose groups (Figure 4B, Supplementary Tables S8-S9).
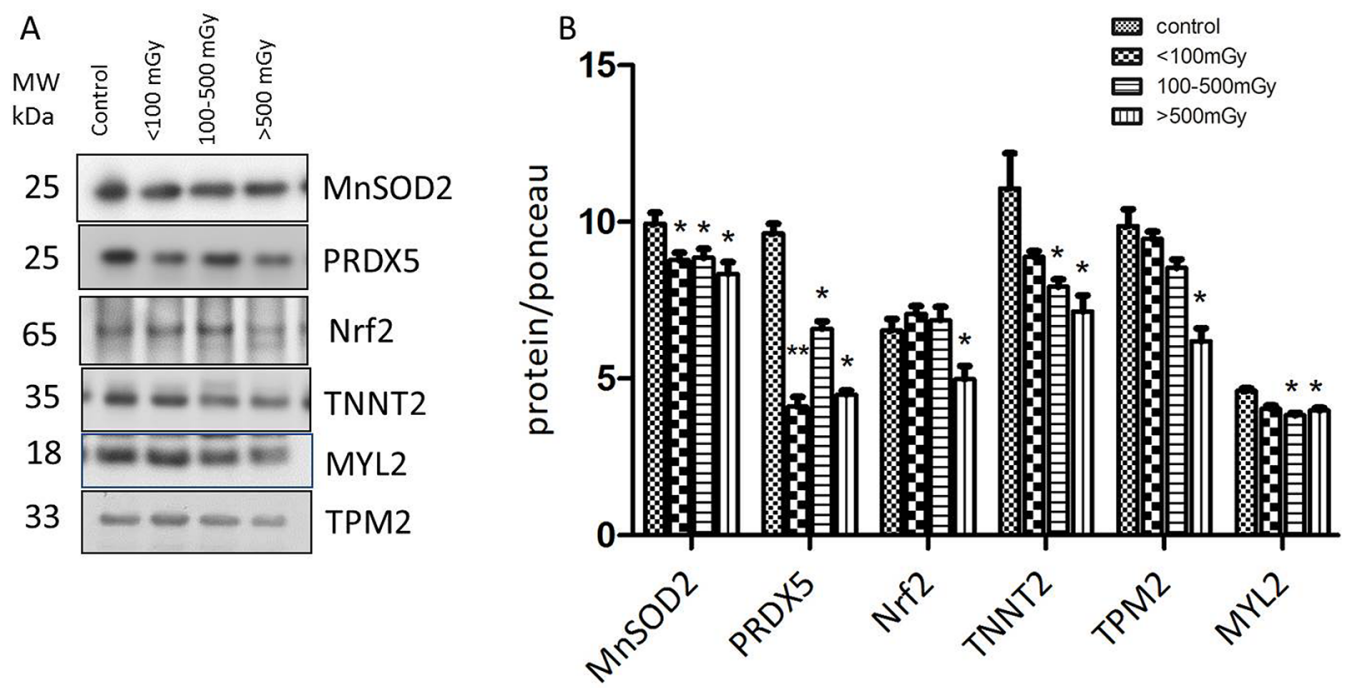

Figure 3: Immunoblot validation of the proteomics data. The heart protein lysates from each individual sample were pooled within the dose groups and tested using anti-Troponin T (TNNT2), anti-Tropomyosin 2 (TPM2), anti- Myosin light chain (MYL2), antiMn superoxide dismutase (SOD2), and anti-Peroxiredoxin 5 (PRDX5) (A).The columns represent the average ratios of relative protein expression in control and irradiated samples. The amount of the total protein was measured by Ponceau S staining for accurate comparison between the groups. The error bars represent standard error of the mean $( \pm \mathrm{SEM})(\mathbf{B})\left(t\right.$-test; $\left.{ }^{*} p<0.05,{ }^{* *} p<0.01 ; n=3\right)$.
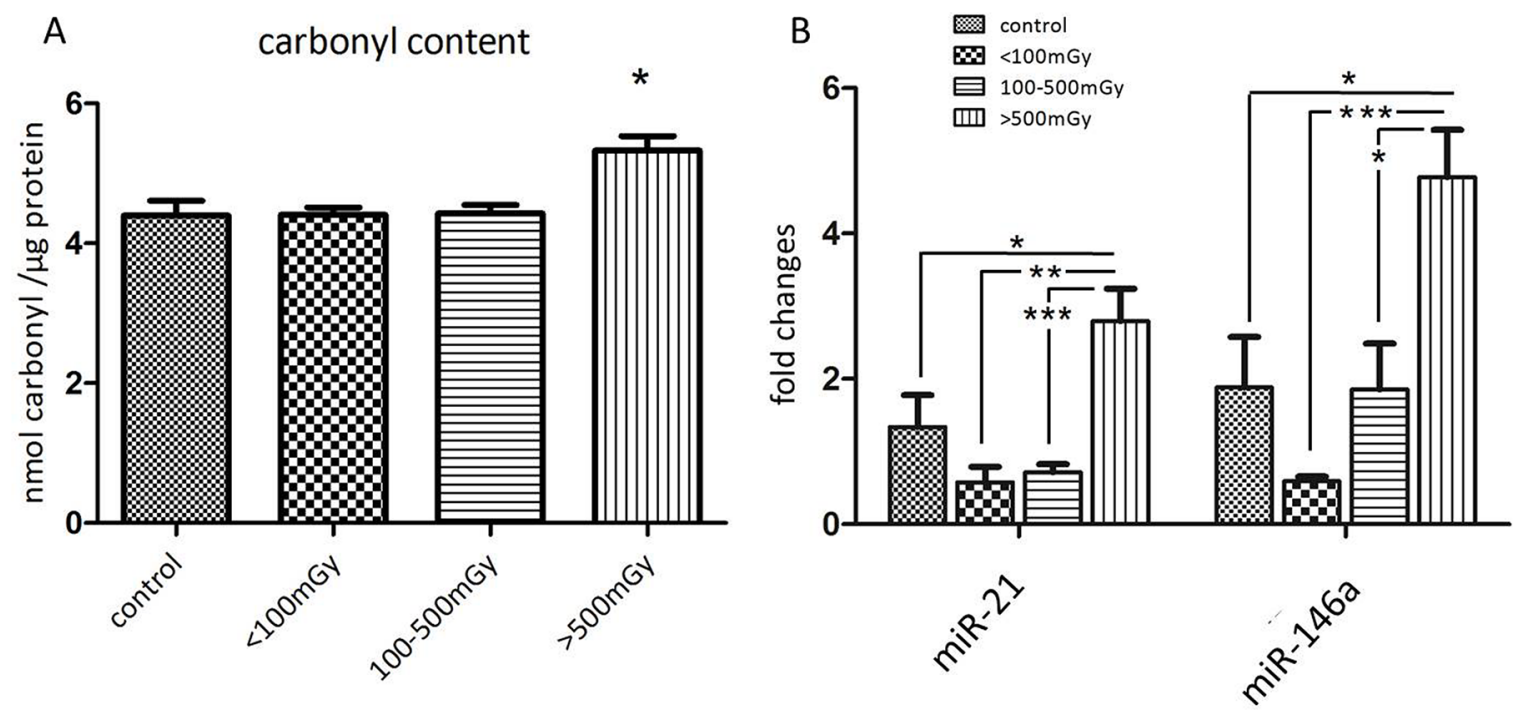

Figure 4: Analysis of the protein carbonyl levels and miR-21 and miR-146a in different dose groups. The total amount of carbonylated protein was measured in individual samples from each dose group. The samples in the control group were run in two technical replicates. Significantly increased level of carbonylated proteins was shown in the dose group $>500 \mathrm{mGy}(\mathbf{A})$. Analysis of miR-21 and miR-146a from samples of all dose groups showed significant upregulation of both miRNAs in the dose group $>500 \mathrm{mGy}(\mathbf{B})$ The error bars represent standard error of the mean $(+\mathrm{SEM})(t$-test; $* p<0.05 ; * * p<0.01 ; * * * p<0.001)$. 


\section{Transcription factor PPAR alpha is inactivated by irradiation}

Analysis of deregulated proteins predicted a significant inactivation of PPAR alpha in all exposed groups (Figure 5A-5C). The number of PPAR alpha target proteins found to have altered expression increased in a dose-dependent manner (Figure 5A-5C). Phosphorylation of PPAR alpha leads to its deactivation in the heart [20]. In agreement with the predicted inactivation, the analysis showed a significant increase in the phosphorylated form of PPAR alpha in pooled samples representing different irradiated groups (Figure 5D). To confirm this at the individual level, the expression of total PPAR alpha and its phosphorylated form were measured separately in all samples (Supplementary Figure S4). The total amount of PPAR alpha was not changed by irradiation (Figure 5E) but there was a significant increase in phospho-PPAR alpha in the irradiated samples in the highest dose groups (Figure 5F).
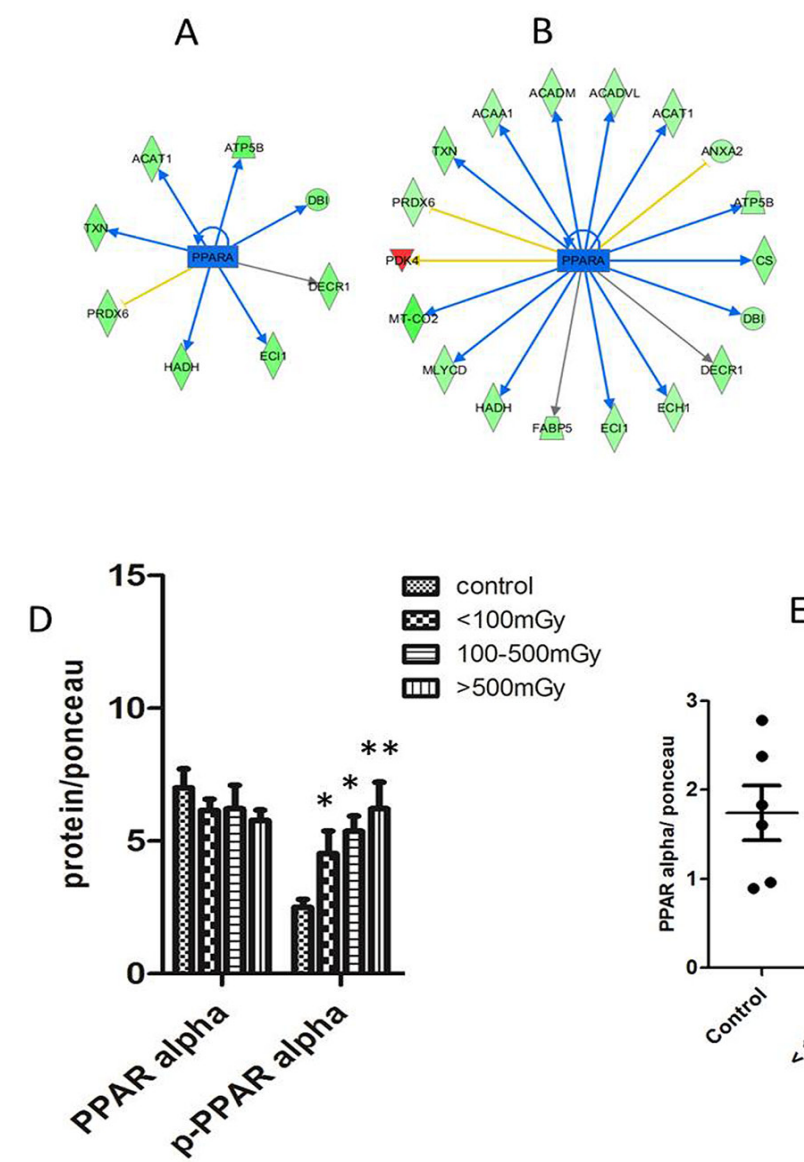

\section{DISCUSSION}

The aim of the present study was to elucidate potential biological mechanisms involved in the radiationinduced IHD in human. For this purpose, we analysed post-mortem samples from the cardiac left ventricle taken from Mayak workers previously exposed to different external radiation doses. This study shows that chronic radiation exposure is able to alter the heart proteome in a dose-dependent manner. The data indicate pronounced radiation-induced changes in proteins involved in the heart function and structure, thereby supporting epidemiological evidence of a significant dose-dependent increase in the IHD incidence reported in the Mayak cohort [2, 3, 21].

In agreement with our previous data obtained in mouse models [12-14, 22, 23] the proteomics analysis shows downregulation of several mitochondrial proteins. The number of deregulated mitochondrial proteins was increased in a dose-dependent manner, indicating increasing mitochondrial dysfunction, a critical pathologic
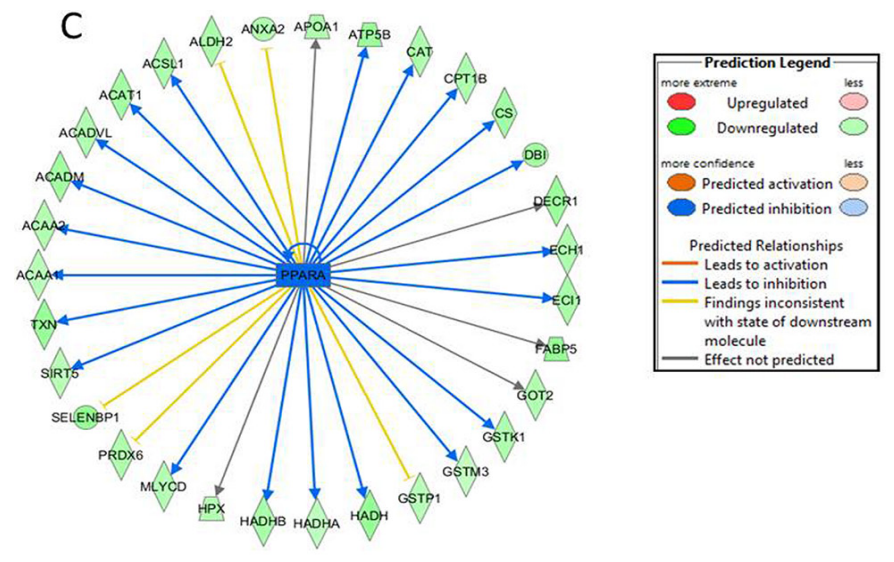

Figure 5: Analysis of the activation status of PPAR alpha. IPA prediction of inactivation of PPAR alpha based on the deregulated proteins from proteomics analysis in the dose groups $<100 \mathrm{mGy}(\mathbf{A}), 100-500 \mathrm{mGy}(\mathbf{B})$ and $>500 \mathrm{mGy}(\mathbf{C})$. The upregulated proteins are marked in red and the down-regulated in green. The blue colour of the node (PPAR alpha) indicates inactivation. The list of proteins is available in Supplementary Tables S2-S4. Immunoblot analysis of total and phospho-PPAR alpha (Ser12) in pooled samples is shown (D). The columns represent the average ratios of relative protein expression in control and irradiated samples. Immunoblot analysis of total PPAR alpha (E) and phospho-PPAR alpha (F) in individual samples from each dose group is shown. The icons represent individual samples in different dose groups. The samples in the control group were run in two technical replicates. The amount of the total protein was confirmed by Ponceau S staining for accurate comparison between the groups $\left(t\right.$-test; ${ }^{*} p<0.05$ ). 
event in IHD [10]. In particular, the expression of proteins belonging to mitochondrial complexes I and III were significantly downregulated. We have previously reported that local heart irradiation in mice induces persistent functional and proteome alterations in cardiac mitochondria that are associated with reduced activity of complexes I and III [13]. Ischemic damage to the heart is also associated with defaults in the activity of complexes I and III $[24,25]$. As the control group used in this study was also suffering from IHD, it can be suggested that chronic radiation worsens the respiratory complex impairment, at least on the proteome level.

A decrease in the mitochondrial respiration rate has been reported to enhance reactive oxygen species (ROS) production [26]. We have shown previously that local heart irradiation permanently increases mitochondrial ROS levels in mice $[12,13]$. This study now shows a dose-dependent decrease in the level of the antioxidant response regulator protein Nrf2. The expression of many Nrf2 target proteins, as well as other proteins of oxidative response, were significantly downregulated (up to $50 \%$ ) in the highest dose group. These included superoxide dismutases (SOD1 and SOD2), peroxiredoxins (1, 2, 3, 5, and 6), glutathione-S-transferases (kappa1, mu2, mu3, omega 1, pil) and catalase. This marked downregulation of antioxidant capacity, coupled with increased carbonylation, suggests an increased radiation-induced ROS production. The expression of miR-146a is increased by oxidative stress [27]. This miRNA was markedly increased in the highest dose group. A target protein of miR-146a is SOD2 [27] which was indeed downregulated in this group.

Mitochondria are not only the main source and but also a target of oxidative damage. As oxidative stressinduced protein modifications such as carbonylation lead to increased protein degradation [28] or inactivation [29], increased oxidation of mitochondrial proteins may explain the vigorous downregulation in their expression seen in this study.

Mitochondria are physically associated with myofibrils, and increased mitochondrial ROS production may lead to impaired contractility through disruption of actin-myosin interactions [30]. Cytoskeletal proteins are considered to be sensitive to redox alterations and the association between oxidative stress and structural damage has been well documented [31]. The oxidation of actin has been shown to result in a strong inhibition of protein polymerisation and in complete disruption of actinfilament organisation [31]. This study shows a significant downregulation of actin isoforms together with many other structural proteins (tubulin, troponin, tropomyosin, desmin and different isoforms of light and heavy myosin). These proteins are major constituents of the contractile apparatus and the severe downregulation seen in the highdose groups may negatively influence cardiac contractility. Cardiac troponin, a sensitive and specific marker for heart damage, is significantly reduced in the two highest dose groups, in comparison to the controls [32].

Prompted by the bioinformatics prediction based mainly on downregulated metabolic and oxidative response proteins, we show here that ionising radiation increases the level of phosphorylation of PPAR alpha and thereby inhibits this transcription factor in a dosedependent manner $[6,20]$. This is in agreement with our previous study showing that high-dose local heart irradiation impairs the cardiac fatty acid oxidation due to inhibition of PPAR alpha activity in mice [14]. The PPAR alpha pathway has been shown to influence antioxidant response and myofibrillar structure [33]. Increased oxidative stress and reduced contractility due to oxidation of myosin have been reported in PPAR alpha knockout mice [34]. We suggest that inactivation of PPAR alpha following radiation exposure adds to the oxidative stress and structural impairment phenotype observed in irradiated hearts.

The level of PPAR alpha and that of several mitochondrial proteins is regulated by mir-21 in different cells and tissues [35-37]. We found significant upregulation in the expression of miR-21 in the left ventricle samples of the highest dose group. This corresponds to the observed downregulation of mitochondrial proteins. Increased levels of mir-21 in heart failure and ischemia have been reported [19, 38, 39].

\section{MATERIALS AND METHODS}

\section{Samples}

Biological samples were collected post-mortem from donors who had previously given informed consent to participate in the study and who had consented to the processing of their personal data in accordance with the Russian Federal Laws No 323-FL of 27.09.2013 and No 261-FL of 25.07.2011. The study was approved by the Southern Urals Biophysics Institute's Institutional Review Board.

The individuals were male Mayak plutonium enrichment plant workers who were exposed only to external gamma rays. The control subjects were nonMayak workers living in the same area. All participants were diagnosed multiple times with IHD during their lifetime and the primary cause of death was IHD. Workers exposed to internal plutonium (Pu alpha-activity in urine $>$ $0.5 \mathrm{kBq}$ ), or who had been diagnosed with cancer or other major somatic diseases were excluded from the study.

All individuals were placed in a cold-room $\left(+4^{\circ} \mathrm{C}\right)$ immediately after the death (approximately within $1 \mathrm{~h}$ ). All autopsies were performed within first 12-24 h after the death. The cardiac left ventricle was collected at autopsy and immediately frozen. Heart tissues from 29 individuals were allocated between four dose groups as follows: 3 individuals to the control group (0 Gy), 6 to 
the dose group $<100 \mathrm{mGy}, 10$ individuals to the group receiving doses between $100-500 \mathrm{mGy}$, and 10 individuals to the dose group $>500$ mGy (Supplementary Table S10). The smoking status and index, alcohol consumption and body mass index of each individual is indicated in Supplementary Table S10.

To expand the number of participants, formalinfixed paraffin-embedded (FFPE) samples from 15 donors were used for miRNA analysis as follows: 3 individuals from control group, 3 individuals from the dose group $<100 \mathrm{mGy}, 4$ individuals from the group representing doses between 100-500 mGy, and 5 individuals from the dose group > $500 \mathrm{mGy}$ (Supplementary Table S10). Four participants $(5,16,26$ and 27) were donors of both frozen and FFPE samples.

\section{Protein extraction}

Frozen heart samples were lysed as described previously [14]. Cardiac left ventricle was ground to a fine powder with a cold $\left(-20^{\circ} \mathrm{C}\right)$ mortar and pestle before being suspended in lysis buffer (SERVA) [14]. Protein concentration was determined by the Bradford assay following the manufacturer's instructions (Thermo Fisher).

\section{Protein purification and mass spectrometry}

Protein lysates $(10 \mu \mathrm{g})$ were digested using a modified filter-aided sample preparation (FASP) protocol [40]. Briefly, the samples were reduced with $10 \mathrm{mM}$ DTT at $60^{\circ} \mathrm{C}$ for $30 \mathrm{~min}$, followed by alkylation with 15 $\mathrm{mM}$ iodoacetamide for $30 \mathrm{~min}$ at room temperature [40]. Samples were diluted using $8 \mathrm{M}$ urea in $0.1 \mathrm{M}$ Tris $/ \mathrm{HCl}$, $\mathrm{pH}$ 8.5, and centrifuged using a $30 \mathrm{kDa}$ cut-off filter (Pall Corporation). After washing with $8 \mathrm{M}$ urea in $0.1 \mathrm{M}$ Tris/ $\mathrm{HCl}, \mathrm{pH} 8.5$, and with $50 \mathrm{mM}$ ammonium bicarbonate $(\mathrm{ABC})$, the proteins were initially digested on the filter with $1 \mu \mathrm{g}$ Lys-C (Wako Chemicals $\mathrm{GmbH}$ ) in $50 \mathrm{mM} \mathrm{ABC}$ at room temperature, followed by addition of $2 \mu \mathrm{g}$ trypsin (Promega) and digestion overnight at $37^{\circ} \mathrm{C}$. Tryptic peptides were collected by centrifugation and acidified with trifluoric acid (TFA) to a $\mathrm{pH}$ of 2.0 . Samples were stored at $-20^{\circ} \mathrm{C}$.

Prior to LC-MS/MS analysis the samples were centrifuged $(16,000 \mathrm{~g})$ for $5 \mathrm{~min}$ at $4^{\circ} \mathrm{C}$. Each sample $(0.5 \mu \mathrm{g})$ representing one donor was analysed separately on a LTQ OrbitrapXL (Thermo Fisher Scientific) coupled to Ultimate 3000 nano-HPLC (Dionex) as described previously [41].

\section{Label-free quantification}

The raw files of the individual measurements were loaded to the Progenesis QI software and analysed as described previously [42, 43]. Briefly, peptide features in the individual runs were aligned in order to reach a maximum overlay of at least $85 \%$. After feature detection, the singly charged features and features with charges higher than +7 were excluded. The samples were grouped according to the radiation dose as described above. Protein identification was performed using the Mascot search engine (Matrix Science, version 2.5.0) with the Ensembl Human database (version 68, 40047886 residues, 105288 sequences).

The following search parameters were used: 10 ppm peptide mass tolerance and 0.6 Da fragment mass tolerance, one missed cleavage was allowed, carbamidomethylation (C) was set as fixed modification, and oxidation $(\mathrm{M})$ and deamidation $(\mathrm{N}, \mathrm{Q})$ were allowed as variable modifications. Search results were reimported into the Progenesis QI software and the resulting summed normalised abundances of unique peptides for every single protein were used for the calculation of abundance ratios and statistical analysis (Student's $t$-test).

A principal component analysis (PCA) was performed using Progenesis QI software (http://www. nonlinear.com), based on all features with charges +2 to +7 resulting in the $\mathrm{PC} 1$ of $23.65 \%$ and $\mathrm{PC} 2$ of $8.36 \%$.

For final quantifications, proteins with ratios greater than 1.30-fold or less than 0.77 -fold ( $t$-test; $p \leq 0.05$ ) were defined as being significantly differentially expressed. The FDR (q value) calculation was used to adjust $p$-values $[44,45]$. The calculation was performed using modified BenjaminiHochberg created by Manuel Weinkauf (https://marum.de/Software_and_Programs. html), licensed under a Creative Commons AttributionNonCommercial-ShareAlike 3.0 Unported License (http:// creativecommons.org/licenses/by-nc-sa/3.0/deed.en_GB). All $p$-values below the corrected significance level q were considered to represent significant results.

\section{Protein-protein interaction and signalling network}

For deregulated proteins, protein-protein interaction and signalling networks were analysed by the software tool INGENUITY Pathway Analysis (IPA) (http://www. INGENUITY.com) [46] and the search tool STRING version 10 (http://string-db.org), coupled to the Reactome database (http://www.reactome.org) [47].

\section{Immunoblot analysis}

Protein lysates from pooled or individual (total and phospho-PPAR alpha) samples were analysed by immunoblotting. For pooled samples, similar amount of protein from each individual belonging to the same radiation dose group (control, < 100 mGy, 100-500 mGy and $>500 \mathrm{mGy}$ ) was combined into a batch representing that group. Proteins separated by $4-12 \%$ SDS-PAGE were transferred to nitrocellulose membranes (GE Healthcare) using a TE 77 semidry blotting system (GE Healthcare) at $1 \mathrm{~mA} / \mathrm{cm}$ for $1 \mathrm{~h}$. The membranes were blocked using $3 \% \mathrm{BSA}$ in TBS, pH 7.4, for $1 \mathrm{~h}$ at room temperature, washed three times in $10 \mathrm{mM}$ Tris- 
$\mathrm{HCl}, \mathrm{pH} 7.4,150 \mathrm{mM} \mathrm{NaCl}$ for $5 \mathrm{~min}$ and incubated overnight at $4{ }^{\circ} \mathrm{C}$ with primary antibodies using dilutions recommended by the manufacturer (Abcam). Immunoblot analysis of heart protein lysate was performed using anti-PPAR alpha (\# ab2779), anti-phospho-PPAR alpha (Ser12)(\# ab3484), anti-troponin T(\# ab156852), antiSOD2 (\# ab13533), anti-peroxiredoxin 5 (\# ab119712), anti-myosin light chain 2 (\# ab 92721), anti-tropomyosin 2 (\# ab96073) and anti-Nrf2 (\# ab31163). After washing three times, the blots were incubated with the appropriate horseradish peroxidase-conjugated or alkaline phosphatase-conjugated anti-mouse, anti-rabbit or antigoat secondary antibody (Santa Cruz Biotechnology) for $2 \mathrm{~h}$ at room temperature and developed using the ECL system (GE Healthcare) or 1-step ${ }^{\mathrm{TM}}$ NBT/BCIP method (ThermoFisher) following standard procedures. Reversible Ponceau staining was used as the loading control. Quantification of digitised images of immunoblot bands was done using ImageJ (http://rsbweb.nih.gov/ij/). Three technical replicates were performed from each pooled sample. For individual analysis, the samples in the control group were run in two technical replicates, all others in one technical replicate.

\section{Protein carbonylation analysis}

To detect the level of protein oxidation, protein carbonylation was measured using the assay kit (Biovision) according to the manufacturer's instructions.

\section{RNA isolation from FFPE blocks and TaqMan- miRNA assays}

Heart tissue was immediately fixed in $4 \%$ buffered formalin for $24 \mathrm{~h}$ and dehydrated with a graded series of ethanol before embedding in paraffin. FFPE blocks were stored in the dark at room temperature. For miRNA analysis, multiple $10 \mu \mathrm{m}$ sections were cut after initial trimming to remove air exposed surfaces. Total RNA was isolated using phenol chloroform gradient as described previously [48] and quantified using NanoDrop spectrophotometer (PeqLab Germany).

Quantitative PCR (Applied Biosystems, Forster City, CA, USA) was performed to analyse the expression of miR-21 (\# 4427975, assay id 000397) and miR-146a (\#4427975, assay id 000468) with the StepOnePlus Detection System (Applied Biosystems, Foster City, CA) according to the manufacturer's instructions. Relative expression values of each miRNA were calculated using the $2-\Delta \Delta \mathrm{CT}$ method, normalised to the control miRNA RNU44 (\# 4427975, assay id 001094) as described earlier [49]. Relative expression values from control and exposed groups were used for further calculations. All samples were analysed at least in duplicate.

\section{Statistical analysis}

Comparative analysis of the data was carried out using the Student's $t$-test (two-paired and unpaired). The significance levels were $* p<0.05(5 \%) ; * *<0.01(1 \%)$ and $* * * p<0.001(0.1 \%)$. The error bars represent the standard error of the mean $( \pm$ SEM).

\section{Data availability}

The raw MS data can be accessed from the RBstore database http://www.storedb.org/store_v3/study. jsp?studyId=1038.

\section{CONCLUSIONS}

This data emphasizes the critical role of PPAR alpha and defect fatty acid oxidation in the radiationinduced IHD. Furthermore, the reduced energy flow from beta oxidation may not be compensated with increased uptake of glucose as the majority of the enzymes in the glycolysis pathway were also downregulated in a dosedependent manner. This may lead to severe ATP depletion in the irradiated heart. Improving the function of PPAR alpha may serve as a useful preventive tool in radiationinduced IHD.

\section{ACKNOWLEDGMENTS AND FUNDING}

This work was supported by grants from Federal Office for Radiation Protection (BfS) (\# 3611S30022), from the European Community's Seventh Framework Program (EURATOM) (\# 295823 PROCARDIO), and Federal Medical Biological Agency (FMBA Russia). We thank Fabian Gruhn, Katrin Linder, Olga Teplyakova and Stefanie Winkler for technical assistance.

\section{CONFLICTS OF INTEREST}

The authors declare no conflicts of interest.

\section{REFERENCES}

1. Azizova TV, Muirhead CR, Druzhinina MB, Grigoryeva ES, Vlasenko EV, Sumina MV, O'Hagan JA, Zhang W, Haylock RG, Hunter N. Cardiovascular diseases in the cohort of workers first employed at Mayak PA in 1948-1958. Radiat Res. 2010; 174:155-168.

2. Azizova TV, Grigoryeva ES, Haylock RG, Pikulina MV, Moseeva MB. Ischaemic heart disease incidence and mortality in an extended cohort of Mayak workers first employed in 1948-1982. Br J Radiol. 2015; 88:20150169.

3. Simonetto C, Azizova TV, Grigoryeva ES, Kaiser JC, Schollnberger H, Eidemuller M. Ischemic heart disease 
in workers at Mayak PA: latency of incidence risk after radiation exposure. PLoS One. 2014; 9:e96309.

4. Yamada M, Wong FL, Fujiwara S, Akahoshi M, Suzuki G. Noncancer disease incidence in atomic bomb survivors, 1958-1998. Radiat Res. 2004; 161:622-632.

5. Stanley WC, Hoppel CL. Mitochondrial dysfunction in heart failure: potential for therapeutic interventions? Cardiovasc Res. 2000; 45:805-806.

6. Finck BN. The PPAR regulatory system in cardiac physiology and disease. Cardiovasc Res. 2007; 73:269-277.

7. Lopaschuk GD, Opie LH. Introduction to JMCC symposium on myocardial energy metabolism in health and disease. $\mathrm{J}$ Mol Cell Cardiol. 2002; 34:1075-1076.

8. Walters AM, Porter GA, Jr., Brookes PS. Mitochondria as a drug target in ischemic heart disease and cardiomyopathy. Circ Res. 2012; 111:1222-1236.

9. Lehman JJ, Kelly DP. Gene regulatory mechanisms governing energy metabolism during cardiac hypertrophic growth. Heart Fail Rev. 2002; 7:175-185.

10. Fillmore N, Mori J, Lopaschuk GD. Mitochondrial fatty acid oxidation alterations in heart failure, ischaemic heart disease and diabetic cardiomyopathy. Br J Pharmacol. 2014; 171:2080-2090.

11. Barger PM, Kelly DP. PPAR signaling in the control of cardiac energy metabolism. Trends Cardiovasc Med. 2000; 10:238-245.

12. Barjaktarovic Z, Schmaltz D, Shyla A, Azimzadeh O, Schulz S, Haagen J, Dorr W, Sarioglu H, Schafer A, Atkinson MJ, Zischka H, Tapio S. Radiation-induced signaling results in mitochondrial impairment in mouse heart at 4 weeks after exposure to X-rays. PLoS One. 2011; 6:e27811.

13. Barjaktarovic Z, Shyla A, Azimzadeh O, Schulz S, Haagen J, Dorr W, Sarioglu H, Atkinson MJ, Zischka H, Tapio S. Ionising radiation induces persistent alterations in the cardiac mitochondrial function of C57BL/6 mice 40 weeks after local heart exposure. Radiother Oncol. 2013; 106:404-410.

14. Azimzadeh O, Sievert W, Sarioglu H, Yentrapalli R, Barjaktarovic Z, Sriharshan A, Ueffing M, Janik D, Aichler M, Atkinson MJ, Multhoff G, Tapio S. PPAR Alpha: A Novel Radiation Target in Locally Exposed Mus musculus Heart Revealed by Quantitative Proteomics. J Proteome Res. 2013; 12:2700-2714.

15. Breckenridge R. Heart failure and mouse models. Dis Model Mech. 2010; 3:138-143.

16. Azizova TV, Day RD, Wald N, Muirhead CR, O'Hagan JA, Sumina MV, Belyaeva ZD, Druzhinina MB, Teplyakov, II, Semenikhina NG, Stetsenko LA, Grigoryeva ES, et al. The "clinic" medical-dosimetric database of Mayak production association workers: structure, characteristics and prospects of utilization. Health Phys. 2008; 94:449-458.

17. Schulte C, Zeller T. microRNA-based diagnostics and therapy in cardiovascular disease-Summing up the facts. Cardiovasc Diagn Ther. 2015; 5:17-36.
18. Bronze-da-Rocha E. MicroRNAs expression profiles in cardiovascular diseases. Biomed Res Int. 2014; 2014:985408.

19. Thum T, Gross C, Fiedler J, Fischer T, Kissler S, Bussen M, Galuppo P, Just S, Rottbauer W, Frantz S, Castoldi M, Soutschek J, Koteliansky V, et al. MicroRNA-21 contributes to myocardial disease by stimulating MAP kinase signalling in fibroblasts. Nature. 2008; 456:980-984.

20. Barger PM, Brandt JM, Leone TC, Weinheimer CJ, Kelly DP. Deactivation of peroxisome proliferator-activated receptor-alpha during cardiac hypertrophic growth. J Clin Invest. 2000; 105:1723-1730.

21. Azizova TV, Muirhead CR, Moseeva MB, Grigoryeva ES, Vlasenko EV, Hunter N, Haylock RG, O'Hagan JA. Ischemic heart disease in nuclear workers first employed at the Mayak PA in 1948-1972. Health Phys. 2012; 103:3-14.

22. Azimzadeh O, Scherthan H, Sarioglu H, Barjaktarovic Z, Conrad M, Vogt A, Calzada-Wack J, Neff F, Aubele M, Buske C, Atkinson MJ, Tapio S. Rapid proteomic remodeling of cardiac tissue caused by total body ionizing radiation. Proteomics. 2011; 11:3299-3311.

23. Azimzadeh O, Scherthan H, Yentrapalli R, Barjaktarovic Z, Ueffing M, Conrad M, Neff F, Calzada-Wack J, Aubele M, Buske C, Atkinson MJ, Hauck SM, Tapio S. Label-free protein profiling of formalin-fixed paraffin-embedded (FFPE) heart tissue reveals immediate mitochondrial impairment after ionising radiation. J Proteomics. 2012; 75:2384-2395.

24. Lesnefsky EJ, Chen Q, Slabe TJ, Stoll MS, Minkler PE, Hassan MO, Tandler B, Hoppel CL. Ischemia, rather than reperfusion, inhibits respiration through cytochrome oxidase in the isolated, perfused rabbit heart: role of cardiolipin. Am J Physiol Heart Circ Physiol. 2004; 287:H258-267.

25. Tompkins AJ, Burwell LS, Digerness SB, Zaragoza C, Holman WL, Brookes PS. Mitochondrial dysfunction in cardiac ischemia-reperfusion injury: ROS from complex I, without inhibition. Biochim Biophys Acta. 2006; 1762:223-231.

26. Heather LC, Carr CA, Stuckey DJ, Pope S, Morten KJ, Carter EE, Edwards LM, Clarke K. Critical role of complex III in the early metabolic changes following myocardial infarction. Cardiovasc Res. 2010; 85:127-136.

27. Ji G, Lv K, Chen H, Wang T, Wang Y, Zhao D, Qu L, Li Y. MiR-146a regulates SOD2 expression in $\mathrm{H} 2 \mathrm{O} 2$ stimulated PC12 cells. PLoS One. 2013; 8:e69351.

28. Dukan S, Farewell A, Ballesteros M, Taddei F, Radman M, Nystrom T. Protein oxidation in response to increased transcriptional or translational errors. Proc Natl Acad Sci U S A. 2000; 97:5746-5749.

29. Tai Y, Inoue H, Sakurai T, Yamada H, Morito M, Ide F, Mishima K, Saito I. Protective effect of lecithinized SOD on reactive oxygen species-induced xerostomia. Radiat Res. 2009; 172:331-338.

30. Bayeva M, Ardehali H. Mitochondrial dysfunction and oxidative damage to sarcomeric proteins. Curr Hypertens Rep. 2010; 12:426-432. 
31. Dalle-Donne I, Rossi R, Giustarini D, Gagliano N, Lusini L, Milzani A, Di Simplicio P, Colombo R. Actin carbonylation: from a simple marker of protein oxidation to relevant signs of severe functional impairment. Free Radic Biol Med. 2001; 31:1075-1083.

32. Skeik N, Patel DC. A review of troponins in ischemic heart disease and other conditions. Int J Angiol. 2007; 16:53-58.

33. Lee WS, Kim J. Peroxisome Proliferator-Activated Receptors and the Heart: Lessons from the Past and Future Directions. PPAR Res. 2015; 2015:271983.

34. Guellich A, Damy T, Lecarpentier Y, Conti M, Claes V, Samuel JL, Quillard J, Hebert JL, Pineau T, Coirault C. Role of oxidative stress in cardiac dysfunction of PPARalpha ${ }^{-/}$ mice. Am J Physiol Heart Circ Physiol. 2007; 293:H93-H102.

35. Zhou J, Wang KC, Wu W, Subramaniam S, Shyy JY, Chiu JJ, Li JY, Chien S. MicroRNA-21 targets peroxisome proliferators-activated receptor-alpha in an autoregulatory loop to modulate flow-induced endothelial inflammation. Proc Natl Acad Sci U S A. 2011; 108:10355-10360.

36. Kida K, Nakajima M, Mohri T, Oda Y, Takagi S, Fukami T, Yokoi T. PPARalpha is regulated by miR-21 and miR-27b in human liver. Pharm Res. 2011; 28:2467-2476.

37. Gomez IG, MacKenna DA, Johnson BG, Kaimal V, Roach AM, Ren S, Nakagawa N, Xin C, Newitt R, Pandya S, Xia TH, Liu X, Borza DB, et al. Anti-microRNA-21 oligonucleotides prevent Alport nephropathy progression by stimulating metabolic pathways. J Clin Invest. 2015; 125:141-156.

38. Cheng Y, Zhang C. MicroRNA-21 in cardiovascular disease. J Cardiovasc Transl Res. 2010; 3:251-255.

39. Schroen B, Heymans S. Small but smart--microRNAs in the centre of inflammatory processes during cardiovascular diseases, the metabolic syndrome, and ageing. Cardiovasc Res. 2012; 93:605-613.

40. Wisniewski JR, Zougman A, Nagaraj N, Mann M. Universal sample preparation method for proteome analysis. Nat Methods. 2009; 6:359-362.

41. Azimzadeh O, Sievert W, Sarioglu H, Merl-Pham J, Yentrapalli R, Bakshi MV, Janik D, Ueffing M, Atkinson MJ, Multhoff G, Tapio S. Integrative proteomics and targeted transcriptomics analyses in cardiac endothelial cells unravel mechanisms of long-term radiation-induced vascular dysfunction. J Proteome Res. 2015; 14:1203-1219.

42. Hauck SM, Dietter J, Kramer RL, Hofmaier F, Zipplies JK, Amann B, Feuchtinger A, Deeg CA, Ueffing M. Deciphering membrane-associated molecular processes in target tissue of autoimmune uveitis by label-free quantitative mass spectrometry. Mol Cell Proteomics. 2010; 9:2292-2305.

43. Merl J, Ueffing M, Hauck SM, von Toerne C. Direct comparison of MS-based label-free and SILAC quantitative proteome profiling strategies in primary retinal Muller cells. Proteomics. 2012; 12:1902-1911.

44. Chen JJ, Wang SJ, Tsai CA, Lin CJ. Selection of differentially expressed genes in microarray data analysis. Pharmacogenomics J. 2007; 7:212-220.

45. Storey JD, Tibshirani R. Statistical significance for genomewide studies. Proc Natl Acad Sci U S A. 2003; 100:9440-9445.

46. Wu J, Liu W, Bemis A, Wang E, Qiu Y, Morris EA, Flannery CR, Yang Z. Comparative proteomic characterization of articular cartilage tissue from normal donors and patients with osteoarthritis. Arthritis Rheum. 2007; 56:3675-3684.

47. D'Eustachio P. Reactome knowledgebase of human biological pathways and processes. Methods Mol Biol. 2011; 694:49-61.

48. Ludyga N, Grunwald B, Azimzadeh O, Englert S, Hofler H, Tapio S, Aubele M. Nucleic acids from long-term preserved FFPE tissues are suitable for downstream analyses. Virchows Arch. 2012; 460:131-140.

49. Anastasov N, Hofig I, Vasconcellos IG, Rappl K, Braselmann H, Ludyga N, Auer G, Aubele M, Atkinson MJ. Radiation resistance due to high expression of miR-21 and G2/M checkpoint arrest in breast cancer cells. Radiat Oncol. 2012; 7:206. 